\title{
Classification of CHD7 Rare Variants in Chinese Congenital Hypogonadotropic Hypogonadism Patients and Analysis of Their Clinical Characteristics
}

Bang Sun, Xi Wang, Jiangfeng Mao, Zhiyuan Zhao, Wei Zhang, Min Nie* and Xueyan Wu*

NHC Key Laboratory of Endocrinology (Peking Union Medical College Hospital), Department of Endocrinology, Peking Union Medical College Hospital, Peking Union Medical College, Chinese Academy of Medical Sciences, Beijing, China

Purpose: $C H D 7$ rare variants can cause congenital hypogonadotropic hypogonadism $(\mathrm{CHH})$ and $\mathrm{CHARGE}$ syndrome. We aimed to summarize the genotype and phenotype characteristics of $\mathrm{CHH}$ patients with $\mathrm{CHD7}$ rare variants.

Edited by:

Corrado Romano,

University of Catania, Italy

Reviewed by:

Paul Lasko,

McGill University, Canada

Jia-Da Li,

Central South University, China

*Correspondence: Min Nie

nm_pumch@aliyun.com

Xueyan Wu

wsheyan@vip.sina.com

Specialty section:

This article was submitted to Genetics of Common and Rare

Diseases,

a section of the journal

Frontiers in Genetics

Received: 11 September 2021 Accepted: 24 November 2021 Published: 03 January 2022

Citation:

Sun B, Wang $X$, Mao J, Zhao Z, Zhang W, Nie $M$ and Wu $X$ (2022) Classification of $\mathrm{CHD} 7$ Rare Variants in

Chinese Congenital

Hypogonadotropic Hypogonadism

Patients and Analysis of Their

Clinical Characteristics.

Front. Genet. 12:770680.

doi: 10.3389/fgene.2021.770680
Methods: Rare sequencing variants (RSVs) were detected by Sanger sequencing in a series of $327 \mathrm{CHH}$ patients and were interpreted and grouped according to the American College of Medical Genetics and Genomics (ACMG) guideline. Detailed phenotyping and genotype-phenotype correlation were analyzed.

Results: The RSV detection rate was 11.01\% (36/327) in the $\mathrm{CHH}$ patients. We identified 30 RSVs and 19 of them were novel. Following ACMG criteria, three variants were pathogenic $(\mathrm{P}), 4$ were likely pathogenic (LP), 3 were of uncertain significance with paradoxical evidence (US1), and 20 were of uncertain significance without enough evidence (US2). All patients (4/4, 100\%) with P or LP variants manifested extragonadal symptoms.

Conclusion: Addition of 19 novel CHD7 variants expanded the spectrum of variants, and pathogenic or likely pathogenic RSVs were more likely to cause syndromic $\mathrm{CHH}$. For $\mathrm{CHH}$ patients carrying $\mathrm{CHD} 7 \mathrm{RSV}$ s, detailed genotyping and phenotyping can facilitate clinical diagnosis and therapy.

Keywords: congenital hypogonadotropic hypogonadism, CHARGE syndrome, CHD7 variants, variant spectrum, phenotype spectrum

\section{INTRODUCTION}

The hypothalamic-pituitary-gonadal axis is indispensable to human puberty and fertility (RiecherRössler, 2017; Herbison, 2016). A deficiency of gonadotropin-releasing hormone (GnRH) results in congenital hypogonadotropic hypogonadism ( $\mathrm{CHH}$, OMIM 146110) - a rare genetic disorder [1 in 30,000 males and 1 in 125,000 females (Laitinen et al., 2011)] featuring incomplete or absent puberty and infertility. If anosmia co-exists, which occurs in about 50\% cases (Bhagavath et al., 2006; Seminara et al., 1998; Stamou and Georgopoulos, 2018; Seminara et al., 1998; Bhagavath et al., 2006; Stamou and Georgopoulos, 2018), the condition is described as Kallmann syndrome (KS), otherwise 
it is considered normosmic congenital hypogonadotropic hypogonadism (nCHH). The clinical and genetic manifestations of $\mathrm{CHH}$ are heterogeneous; more than 40 pathogenic genes have been identified, accounting for about $50 \%$ of the pathogenesis (Seminara et al., 1998; Bhagavath et al., 2006; Stamou and Georgopoulos, 2018).

The CHD7 gene, which is located in chromosome 8q12 and spans $188 \mathrm{~kb}$ (Vissers et al., 2004), consists of 38 exons and encodes chromodomain helicase DNA binding protein 7 (CHD7), a 2997amino acid protein including nine functional regions. $\mathrm{CHD} 7$ is the first identified chromatin-remodeling protein contributing to human puberty; previous studies have proved its critical role in maintaining GnRH level and olfactory neuron maturation (Kim et al., 2008). Its structure is highly conserved, and it is expressed ubiquitously in the human body. Pathogenic variants of CHD7 exist in about $10 \%$ of $\mathrm{CHH}$ patients, and they are also the major pathogenic cause (detection rate is more than 90\%) for another autosomal dominant disease, CHARGE syndrome (OMIM, 214800) (Bergman et al., 2011). The acronym CHARGE stands for Coloboma of the eye, Heart defects, Atresia of the choanae, Retardation of growth/development, Genital abnormalities, and Ear abnormalities (Martin, 2010). According to Verloes' diagnostic criteria (Verloes, 2005), there are eight key clinical items, including three major items (coloboma, choanal atresia, semicircular canal anomalies) and five minor items (rhombencephalic anomalies, hypothalami-hypophyseal dysfunction, external or middle ear malformations, malformation of mediastinal viscera, and intellectual disability). Based on the number of major and minor items, patients were classified into three groups: typical, partial, and atypical CHARGE. Besides $\mathrm{CHH}$ and CHARGE syndrome related symptoms, CHD7 variants can also lead to other CHD7-related manifestations, including abnormalities of the skeleton, muscle, skin, digestive system and urinary system.

Growth retardation and genital abnormalities are potential overlapping symptoms between CHARGE and CHH. Missense CHD7 variants are more common in CHH patients, whereas null variants (e.g., nonsense, frameshift) are more common in CHARGE syndrome (Balasubramanian et al., 2014). However, the specific correlation between the two diseases has not been clarified owing to the lack of detailed variant annotation and large clinical trials. In this study, we aimed to explore the genotype, phenotype and genotype-phenotype correlation of $\mathrm{CHH}$ patients with $C H D 7$ rare sequencing variants (RSVs). We analyzed $C H D 7$ RSVs in a series of 327 Chinese CHH patients, classified them according to the American College of Medical Genetics and Genomics (ACMG) guideline, evaluated their clinical characteristics (focused on CHARGE-related symptoms and other CHD7-related ones), and performed genotype-phenotype correlation analysis.

\section{METHODS}

\section{Participants and Inclusion/Exclusion Criteria}

Three hundred and fifty-seven probands were admitted to Peking Union Medical College Hospital (Beijing, China) between
January 2005 and December 2012 and diagnosed as CHH. This study included 327 unrelated $\mathrm{CHH}$ Chinese probands: $148 \mathrm{nCHH}$ (141 males; Seven females) and 179 KS (167 males; 12 females), excluding 30 patients diagnosed as carrying FGFR1 variants (Nie et al., 2021). The study was approved by the Ethics Committee for Human Research of Peking Union Medical College Hospital; all patients and guardians of children provided written informed consent.

The inclusion and exclusion criteria were laid down based on $\mathrm{CHH}$ standard consensus (Boehm et al., 2015). Inclusion criteria: (1) absent or incomplete puberty by the age of 18 years for male and 16 years for female; (Herbison, 2016) cryptorchidism without a definite diagnosis; (2) serum testosterone $\leq 100 \mathrm{ng} / \mathrm{dl}$ for males or estradiol $\leq 20 \mathrm{pg} / \mathrm{ml}$ for females, with low or normal serum gonadotropin level. The participants were classified as having KS; (3) when the inquiry information showed the presence of anosmia or hyposmia, otherwise they were classified as having $\mathrm{nCHH}$. Exclusion criteria: diagnosis of secondary hypogonadotropic hypogonadism induced by tumors, trauma, drugs, or other systemic diseases.

\section{Variants Screening}

We collected blood samples from all participants. Focusing on the CHD7 gene, the Sanger sequencing technique was used to detect causative variants. Genomic DNA extraction was performed using the QIAGEN Midi Blood kit (QIAGEN, Germany) from leukocytes in peripheral blood. Polymerase chain reaction (PCR) was used to amplify the specific sequence of $\mathrm{CHD7}$ gene. All experiments followed the manufacturer's instructions.

We read the sequencing results by 4 Peaks (Nucleobytes, Netherlands), then blasted each in NCBI (https://blast.ncbi.nlm. nih.gov/Blast.cgi), against the following reference sequences: NG_007009 (g.DNA); NM_017780 (c.DNA); NP_060250 (p. Protein). Considering the digenic or oligogenic pathogenic possibility, which may explain why individuals with the same CHD7 variant presented with different phenotypes, we screened and annotated the other $\mathrm{CHH}$-related genes by Sanger sequencing, including ANSO1, FGF8, PROK2, PROKR2, GNRH1, GNRHR, KISS1, KISS1R, TAC3, TACR3, LEP, LEPR, NELF, WDR11, HS6ST1, and SEMA3A. All variants were reported according to Human Genome Variation Society (HGVS) nomenclature rules (Dunnen and Antonarakis, 2000).

\section{Annotation for Identified Variants}

Based on the ACMG standards and guidelines ${ }^{3}$, we filtered all detected variants step by step. The core workflow includes general population database search, case-control statistical comparison based on calculation of odds ratio (OR) and the $95 \%$ confidence interval $(95 \% \mathrm{CI})$ (referring to the statistical analysis section for details), disease database search, literature query, variant-typespecific analysis, and computational prediction (Figure 1). We used the allelic frequency from gnom $A D$ database to calculate $\mathrm{OR}$ and define a rare sequencing variant (RSV) [a maximum allele frequency $(\mathrm{MAF})<0.0001]$. Variants databases from patients included ClinVar and CHD7 databases. Literature query was performed in PubMed, Google Scholar and China National Knowledge Infrastructure (CNKI). The search key word 


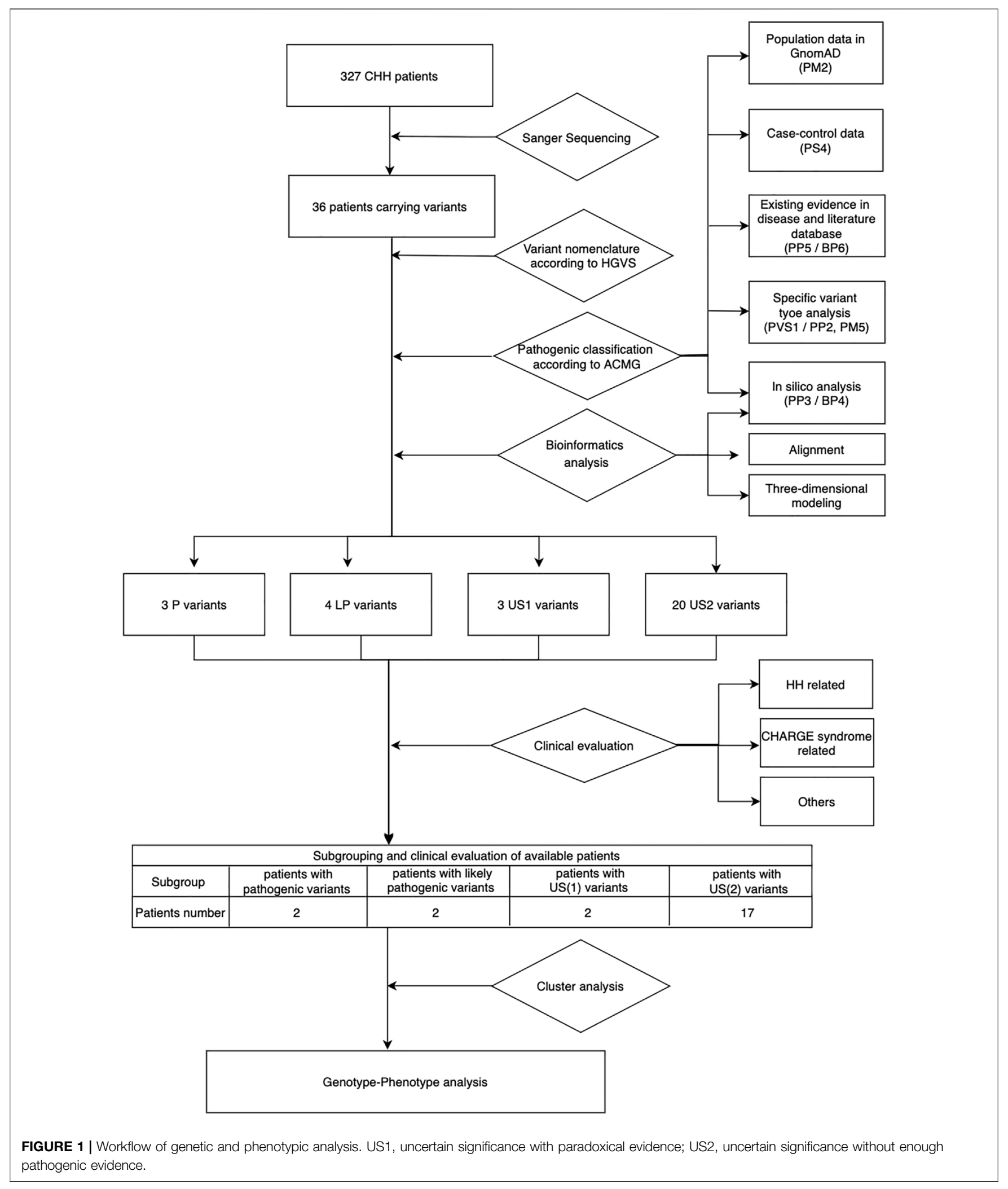

included "CHD7 gene," "hypogonadotropic hypogonadism" and related variants without any language, timeframe or article type restriction.
Computational prediction included three parts: pathogenic prediction by in silico tools, conservation analysis by alignment, and three-dimensional visualization by three-dimensional 
modeling software. In silico tools included five [SIFT (Sim et al., 2012), Polyphen-2 (Adzhubei et al., 2010), SNP\&GO (Schwarz et al., 2010), Mutation Assessor (Reva et al., 2011), and Mutpred (Li et al., 2009)] for missense pathogenicity prediction (when more than three predicted the variants as pathogenic, the evidence pointed to PP3, otherwise it was BP4) and two [Splice Site Score Calculation and SpliceAI (Jaganathan et al., 2019)] for splicing sites (Table 2). We judged the evolutionary conservation of each amino acid site by aligning the human CHD7 sequence with orthologs of other 25 related species in UniProt database with Clustal W; the more conservative the site is, the more important it may be for protein function, which indirectly reveals its pathogenicity. Amino acid change was visualized by ChimeraX, a threedimensional modeling software. By comparing the change in the identity of wild and mutant amino acids and their contact with other amino acids in a spatial model, we can predict the potential pathogenic effects the specific variant brings to the protein.

Data for all pathogenic and benign variants considering the above aspects were collected when available, and then based on the weight of the evidence, we classified the variants into six groups: pathogenic (P), likely pathogenic (LP), uncertain significance with paradoxical evidence (US1), uncertain significance without enough pathogenic evidence (US2), likely benign (LB), and benign (B). All specific criteria above were based on the ACMG guideline, except that we further divided the US ones into US1 and US2 based on the type of evidence (paradoxical evidence or insufficient pathogenic evidence).

\section{Clinical Evaluation}

We recorded basic information of all $\mathrm{CHH}$ patients during their first visit, including sex, age, height, weight, testicular size, penis length, hormone levels, and olfactory magnetic resonance imaging (MRI) results, medical history and family history. Prader orchidometer was used to measure the testicular size, and the mean volume was calculated for statistical analysis. Hormone tests results included levels of serum follicle-stimulating hormone (FSH), luteinizing hormone (LH), and testosterone $(\mathrm{T})$, which were measured by chemiluminescent immunoassays (Bayer Diagnostics Corporation, United States).

For CHH patients with CHD7 RSVs, detailed phenotyping was performed when possible. Clinical evaluation items were based on the Human Phenotype Ontology database and were further classified into three types: CHH-related, CHARGE syndrome-related (including the function and appearance of eyes, nose, ears, mouth, and face; growth status, intelligence, and heart condition), and others (including the condition of the skeleton, muscle, palatal arch, skin, digestive system, and urinary system and synkinesia). The CHARGE-related symptoms were based on Verloes' criteria ${ }^{13}$. Information about bone deformity, limb development, skin texture, condition of the digestive and urinary systems, function and appearance of the eyes and ears, and medication history was collected by inquiry and physical examination. MRI or temporal bone computed tomography (CT) was used to evaluate the middle and inner ear conditions whenever possible along with an auditory test. The condition of the eyes was evaluated by ophthalmologist with fundoscopy. Cardiac ultrasound were performed in cases with clinical suspicion of cardiac abnormalities. Results of genotypic and phenotypic analyses of family members were also collected if available.

\section{Statistics Analysis}

Continuous variables were checked for normal distribution by SPSS version 21 software package (IBM, China). Normally distributed variables are described by the mean and SD, and nonnormally distributed variables are described by the median and interquartile range. Categorical variables are presented as a percentage. The OR and $95 \% \mathrm{CI}$ were calculated by using an online OR calculator (http://www.hutchon.net/ConfidOR. $\mathrm{htm} /$ ) (Bland and Altman, 2000). When OR > 5.0 and the 95\% CI did not include 1.0, it was assumed that the prevalence of the variant in patients is significantly increased compared with the prevalence of controls.

\section{RESULTS}

\section{Patients Harboring CHD7 RSVs}

A total of $36 \mathrm{CHH}(20 \mathrm{KS} ; 15 \mathrm{nCHH})$ out of 327 (11.01\%) carried heterozygous $\mathrm{CHD} 7$ gene RSVs (MAF <0.0001) (Supplementary Figure S1). All these patients complained of puberty absence and small testicular size $[1.5(1,2.75)]$, and the percentage of cryptorchidism and concealed penis were $16.67 \%(6 / 36)$ and $5.56 \%(2 / 36)$, respectively. The mean diagnosis age was $19.75 \pm 4.88$ years. Among 13 patients who accepted the olfactory MRI, all patients had absence or hypoplasia of the olfactory bulb or (and) tract to different degrees. The results of hormone tests, the mean basal LH, FSH, and testosterone levels were consistent with hypogonadotropic hypogonadism, and the specific values are shown in Table $\mathbf{1}$.

Thirty CHD7 gene variants were detected, including three splice site (c.2835+1G > A, c. $5210+3 \mathrm{~A}>\mathrm{G}$, and c.7972-1G > C) and 27 missense variants (D3N, Q40H, S137A, D618G, D728N, E738D, D740G, P783S, I872T, R886W, M888V, R897P, R944H, C1251F, I1311T, G1506S, K1699E, R1743C, N2118S, S2123C, K2235Q, R2284Q, V2327G, R2361S, D2390E, S2453N, and N2808K) (Supplementary Figure S1). All variants were distributed throughout the $\mathrm{CHD} 7$ gene and showed a certain cluster in exons 2, 4, and 8. Six variants were located in known functional domains of CHD7 protein: three (D740G, M888V, and R886W) in Chromo2, one $(\mathrm{C} 1251 \mathrm{~F})$ in SNF2, one (I1311T) in Helicase, and one (c.7972$1 \mathrm{G}>\mathrm{C}$ ) in BRK2, and 12 variants clustered in a protein region that was not conserved and its function was unknown (Figure 2).

Particularly, each variant existed only in one proband, except for Q40H, R1743C and E738D presenting in two patients and G1506S in three patients. Three patients harbored other heterozygous missense gene variants besides CHD7: one patient with GNRH1 (G34R in Patient 16) and two patients 
TABLE 1 | Basic information of 36 patients harboring CHD7 rare variants.

\begin{tabular}{|c|c|c|c|c|c|c|c|c|c|c|c|c|c|c|c|}
\hline \multirow[t]{2}{*}{$\begin{array}{l}\text { Patients } \\
\text { No. }\end{array}$} & \multirow[t]{2}{*}{ Dignosis } & \multirow[t]{2}{*}{ Gender } & \multirow{2}{*}{$\begin{array}{l}\text { Age of } \\
\text { dignosis } \\
\text { (year) }\end{array}$} & \multirow[t]{2}{*}{$\begin{array}{l}\text { Olfactory } \\
\text { MRI }\end{array}$} & \multirow{2}{*}{$\begin{array}{l}\text { Basal } \\
\text { testicular } \\
\text { size, } \\
\text { left/right } \\
\text { (cm) }\end{array}$} & \multicolumn{3}{|c|}{$\begin{array}{l}\text { Basal sex hormone } \\
\text { examination }\end{array}$} & \multicolumn{3}{|c|}{ Typical HH } & \multicolumn{4}{|c|}{ Variants } \\
\hline & & & & & & $\begin{array}{c}\text { LH } \\
\text { (basal) } \\
\text { (mlU/L) }\end{array}$ & $\begin{array}{l}\text { FSH } \\
\text { (basal) } \\
\text { (mlU/L) }\end{array}$ & $\begin{array}{c}\mathrm{T} \\
\text { (basal) } \\
\text { (nmoL/ } \\
\text { L) }\end{array}$ & $\begin{array}{l}\text { Cryptorchidism } \\
\text { or Concealed } \\
\text { penis }\end{array}$ & $\begin{array}{l}\text { Delayed } \\
\text { puberty }\end{array}$ & $\begin{array}{c}\text { Primary } \\
\text { amenorrhoea }\end{array}$ & $\begin{array}{l}\text { Nucleotide } \\
\text { change }\end{array}$ & Protein change & Zygosity & $\begin{array}{l}\text { Other } \\
\text { variants }\end{array}$ \\
\hline P1 & KS & M & 24 & NE & NA & 0.3 & 0.01 & 1.71 & - & + & - & c. $2347 \mathrm{C}>\mathrm{T}$ & p.Pro783Ser & Het & - \\
\hline P2 & $\mathrm{nCHH}$ & M & 20 & NE & $3 / 3$ & 0 & 0 & 1.1 & - & + & - & c.6703 A > C & p.Lys2235Gln & Het & - \\
\hline P3 & KS & M & 20 & NE & 2/2 & 0.3 & 1.1 & 0.8 & - & + & - & c. $7972-1 G>C$ & - & Het & - \\
\hline P4 & KS & M & 29 & NE & $5 / 5$ & 0.1 & 0.34 & 3.05 & - & + & - & c.6980 T > G & p.Val2327Gly & Het & - \\
\hline P5 & KS & M & 26 & 1 & 0/3 & 0 & 0.2 & 1.03 & Concealed penis & + & - & c. $7083 \mathrm{G}>\mathrm{C}$ & p.Arg2361Ser & Het & - \\
\hline P6 & KS & M & 14 & 2 & $1 / 1$ & 0.06 & 1.8 & 1.02 & - & + & - & c.3932 T > C & p.lle1311Thr & Het & - \\
\hline P7 & KS & M & 19 & 3 & $3 / 3$ & 0.18 & 0.48 & 2.5 & - & + & - & c.5227 C > T & p.Arg1743Cys & Het & \\
\hline P8 & $\mathrm{nCHH}$ & M & 20 & NE & $6 / 2$ & 2.6 & 0.3 & 1.96 & Cryptorchidism (bilateral) & + & - & $\mathrm{c} .7170 \mathrm{~T}>\mathrm{G}$ & p.Asp2390Glu & Het & - \\
\hline P9 & KS & M & 24 & 3 & $2 / 2$ & 0.04 & 0.7 & - & $\begin{array}{l}\text { Concealed penis } \\
\text { Cryptorchidism (bilateral) }\end{array}$ & + & - & $c .6353 A>G$ & p.Asn2118Ser & Het & - \\
\hline P10 & KS & M & 22 & NE & $2 / 2$ & 0.47 & 2.4 & 0.15 & - & + & - & c. $409 \mathrm{~T}>\mathrm{G}$ & p.Ser137Ala & Het & - \\
\hline P11 & KS & $\mathrm{F}$ & 18 & NE & NA & 1.34 & 2.5 & 39.6 & - & + & - & c. $2214 \mathrm{~A}>\mathrm{C}$ & p.Glu738Asp & Het & - \\
\hline P12 & $\mathrm{nCHH}$ & M & 20 & NE & $1 / 1$ & 0.4 & 1.3 & 0.9 & - & + & - & c. $2831 \mathrm{G}>\mathrm{A}$ & p.Arg944His & Het & $\begin{array}{l}\text { WDR11, } \\
\text { p.Leu891Trp(Het) }\end{array}$ \\
\hline P13 & KS & M & 20 & NE & $4 / 4$ & 0.47 & 1.03 & 0.97 & - & + & - & C. $120 \mathrm{~A}>\mathrm{C}$ & p.Gln4OHis & Het & - \\
\hline P14 & KS & M & 19 & NE & $1 / 1$ & 0 & 0.4 & 0.88 & - & + & - & $\begin{array}{l}\mathrm{c} .2658 \mathrm{C}>\mathrm{T} \\
\mathrm{c} .4516 \mathrm{G}>\mathrm{A}\end{array}$ & $\begin{array}{l}\text { p.Arg886Trp } \\
\text { p.Gly1506Ser }\end{array}$ & Het & - \\
\hline P15 & $\mathrm{nCHH}$ & $\mathrm{F}$ & 24 & NE & NE & 0 & 0 & 0.99 & - & + & - & c. $2831 \mathrm{G}>\mathrm{A}$ & p.Arg944His & Het & - \\
\hline P16 & KS & M & 8 & NE & $1 / 1$ & 0.04 & 1.2 & 0.49 & Cryptorchidism (right) & + & - & c. $1853 \mathrm{~A}>\mathrm{G}$ & p.Asp618Gly & Het & $\begin{array}{l}\text { GNRH1, } \\
\text { p.Gly34Arg(Het) }\end{array}$ \\
\hline P17 & $\mathrm{nCHH}$ & M & 19 & NE & $2 / 1$ & 1.25 & 1.77 & 0.83 & - & + & - & $c .4516 \mathrm{G}>\mathrm{A}$ & p.Gly1506Ser & Het & - \\
\hline P18 & KS & M & 14 & 1 & $2 / 2$ & 0.06 & 1.5 & 1.36 & - & + & - & $\mathrm{c} .2615 \mathrm{~T}>\mathrm{C}$ & p.lle872Thr & Het & - \\
\hline P19 & KS & M & 28 & NE & $1 / 2$ & 1.17 & 3.5 & 1.36 & - & + & - & $c .3752 G>T$ & p.Cys1251Phe & Het & - \\
\hline P20 & $\mathrm{nCHH}$ & M & 22 & NE & $4 / 4$ & 0.38 & 1.4 & 1.73 & - & + & - & $\begin{array}{c}\text { c. } 2835+1 G> \\
A / G\end{array}$ & - & Het & - \\
\hline P21 & $\mathrm{nCHH}$ & M & 15 & NE & $1 / 1$ & 0 & 0.1 & 7.09 & - & + & - & $\begin{array}{c}c .5210+3 A> \\
A / G\end{array}$ & - & Het & - \\
\hline P22 & KS & M & 12 & 3 & $1 / 1$ & 0 & 0.3 & 0.07 & Cryptorchidism (bilateral) & + & - & c.5095 A > G & p.Lys1699Glu & Het & $\begin{array}{l}\text { WDR11, } \\
\text { p.Val336Phe(Het) }\end{array}$ \\
\hline P23 & $\mathrm{nCHH}$ & M & 21 & NE & $3 / 3$ & 1.64 & 2 & 0.48 & - & + & - & c. $2662 A>G$ & p.Met888Val & Het & - \\
\hline P24 & $\mathrm{nCHH}$ & M & 25 & NE & $0 / 0$ & 0 & 0.07 & 2.85 & Cryptorchidism (bilateral) & + & - & $c .2219 A>G$ & p.Asp740Gly & Het & - \\
\hline P25 & KS & $\mathrm{F}$ & 14 & NE & - & 0.1 & 1.1 & 0.4 & - & + & - & c. $6851 \mathrm{G}>\mathrm{A}$ & p.Arg2284Gin & Het & - \\
\hline P26 & KS & M & 11 & 2 & $1 / 0.5$ & 0.09 & 0.89 & 1.38 & Cryptorchidism (bilateral) & + & - & c. $7 \mathrm{G}>\mathrm{A}$ & p.Asp3Asn & Het & - \\
\hline P27 & $\mathrm{nCHH}$ & M & 20 & NE & $2 / 2$ & 0.42 & 0.8 & 0.46 & - & + & - & c. 2831 G > A & p.Arg944His & Het & - \\
\hline P28 & $\mathrm{nCHH}$ & M & 30 & NE & $8 / 6$ & NA & NA & NA & - & + & - & $c .2690 \mathrm{G}>\mathrm{C}$ & p.Arg897Pro & Het & - \\
\hline P29 & KS & M & 19 & 2 & $1.5 / 1.5$ & 0.22 & 1.3 & 0.89 & - & + & - & c. $8424 \mathrm{C}>\mathrm{A}$ & p.Asn2808Lys & Het & - \\
\hline P30 & KS & M & 17 & NE & $1 / 1$ & 0.01 & 0.44 & 0.62 & - & + & - & $c .2182 \mathrm{G}>\mathrm{A}$ & p.Asp728Asn & Het & - \\
\hline P31 & KS & $\mathrm{F}$ & 18 & 3 & NE & 0 & 0.8 & NA & - & + & + & c.6368 C > G & p.Ser2123Cys & Het & - \\
\hline P32 & KS & M & 23 & 3 & $2 / 1$ & 0 & 0.5 & 1.13 & - & + & - & c. $2214 \mathrm{~A}>\mathrm{C}$ & p.Glu738Asp & Het & - \\
\hline P33 & KS & M & 22 & NE & $2 / 3$ & 1.66 & 2 & 0.12 & - & + & - & $0.7358 \mathrm{G}>\mathrm{A}$ & p.Ser2453Asn & Het & - \\
\hline P34 & KS & M & 19 & 1 & $2 / 1$ & 0 & 0.2 & 0.4 & - & + & - & c. $4516 \mathrm{G}>\mathrm{A}$ & p.Gly1506Ser & Het & - \\
\hline P35 & KS & M & 19 & 1 & $1 / 1$ & 0 & 0.4 & 0.97 & - & + & - & C. $120 \mathrm{~A}>\mathrm{C}$ & p.Gln4OHis & Het & - \\
\hline P36 & KS & M & 16 & 1 & $1 / 1$ & 0 & 0.4 & 13.0 & - & + & - & c.5227 C > T & p.Arg1743Cys & Het & - \\
\hline
\end{tabular}

$n C H H$ normosmic congenital hypogonadotropic hypogonadism: KS, Kallmann syndrome: 1, absence of olfactory bulb and tract, 2, hypoplasia of olfactory bulb and absence of olfactory tract, 3, hypoplasia of olfactory bulb and tract: $M$, male; F, female; NE, no evaluation; +, positive symptoms; -, negative symptoms; NA, not eavauation. 

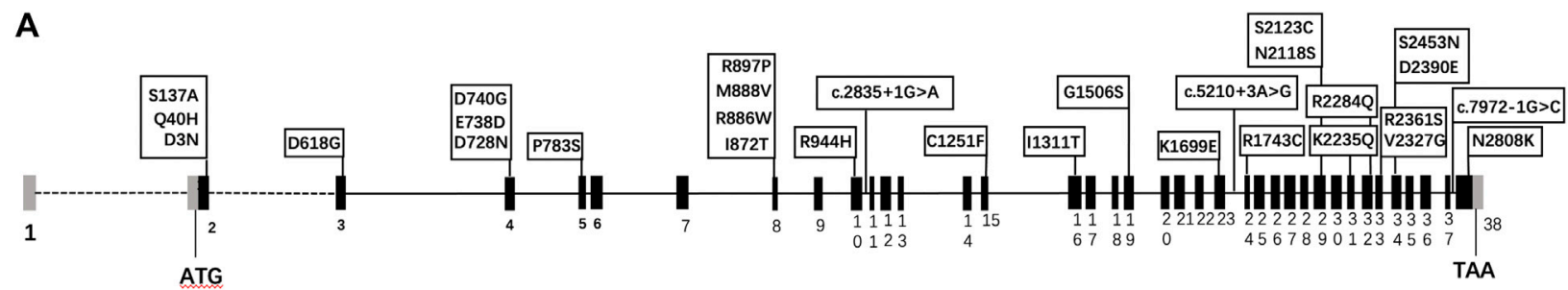

B

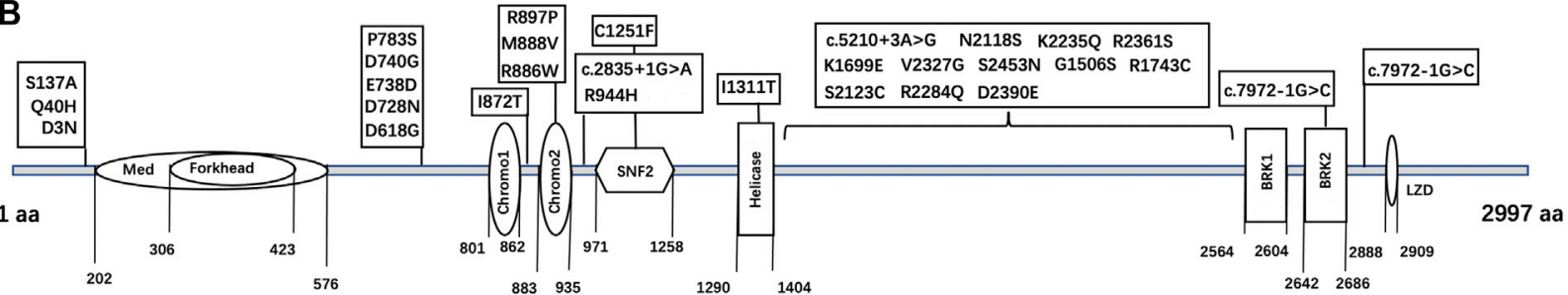

FIGURE 2|The schematic diagram of CHD7 gene (A) and protein (B) structure. Black bars: coding exons; grey bars: non-coding sequences; various shapes in the protein diagram: functional domains of the $\mathrm{CHD} 7$ protein.

with WDR11 (V336F in Patient 22 and L891W in Patient 12) (Table 1).

\section{Classification of Variants Based on the ACMG Guideline and Bioinformatics Analysis}

We classified the $30 \mathrm{CHD} 7$ variants according to HGVS, then analyzed their pathogenicity based on ACMG criteria (Table 2). Besides R944H, which had OR $<5.0$ and 95\% CI included 1.0, the other variants were either absent in the gnomAD database or the corresponding $\mathrm{OR} \geq 5.0$ and $95 \% \mathrm{CI}$ did not include 1.0. Among these 30 variants, 11 were recurrent and 19 were reported for the first time. Six variants existed in the ClinVar database: S137A, P783S and D2390E were reported in CHARGE syndrome patients; $\mathrm{R} 944 \mathrm{H}$ both in CHARGE syndrome and $\mathrm{HH}$ patients; R2284Q had no specific disease description. The amino acid sites of five variants were reported in the $\mathrm{CHD} 7$ variants database: three (c.5210+3 A > G, D728N, and R944H) had the same amino acid change and two (C1251F and R2284Q) mutated into a different one. Besides, four [D728N (Bartels et al., 2010), R886W (Marcos et al., 2014), D740G (Bilan et al., 2012), R944H (Bartels et al., 2010)] were reported by other researchers in $\mathrm{CHH}$ or CHARGE syndrome cohorts, and the detection rates were $1 / 642,1 / 313,1 / 50$, and $1 / 642$, respectively.

In computational pathogenicity prediction, firstly, the results of in silico analyses showed that, except for E738D and K2235Q (less than three tools defined these as pathogenic) with BP4 evidence, the remaining missense variants and three splicing site variants all were pathogenic and showed PP3 evidence. Secondly, focusing on 17 novel missense variants, alignment results indicated that except for $\mathrm{N} 2808 \mathrm{~K}$, the other 16 wild type residues at a specific site were highly conserved across 25 different species (Figure 3). Thirdly, as three-dimensional models of R897P, I1311T, C1251F, R1743C, and K1699E (built by SwissModel) are available, we can see the direct harmful effect of a single amino acid change in the tertiary structure of the protein. The size, charge, and hydrophobicity of all five residues changed at the specific site: for R897P, the acidic amino acid changed into a nonpolar aliphatic one. Seven old contacts and 1 hydrogen bond (H-bond) broke, which accompanied with 6 new contacts and 1 clashes formed; for $\mathrm{C} 1251 \mathrm{~F}$, the neutral polar amino acid changed to an aromatic one, besides, 1 old contacts, 1 clashes (unfavorable interactions where atoms are too close together) and $1 \mathrm{H}$-bond lost; for I1311T, a neutral polar one replaced the nonpolar aliphatic amino acid, and 3 old contacts broke; for K1699E, 8 new contracts formed; for R1743C, 7 contacts lost (Figure 4). Based on these findings, 3/30 (10.00\%) variants were classified as $\mathrm{P}$ variants, $4 / 30(13.33 \%)$ were LP, 3/30 (10.00\%) were US1, and 20/30 (66.67\%) were US2.

None of the second variant could be classified as the digenic pathogenic one according to the existing evidence. GNRH1 variants were inherited in an autosomal recessive manner; therefore, a direct pathogenic effect was impossible. WDR11 variants, which showed an autosomal dominant pattern of inheritance, had V336F classified as US1 and L891W as US2. Owing to the lack of experimental evidence, it is necessary to study whether these variants contribute to disease pathogenesis in a synergistic manner.

\section{Clinical Evaluation and Genotype-Phenotype Correlation}

Twenty-three patients (16 KS and seven $\mathrm{nCHH} ; 21$ male and two female) harboring RSVs accepted detailed clinical evaluation, including two with $\mathrm{P}$ variant, two with $\mathrm{LP}$ variant, two harboring US1, and 17 with US2 (Table 3). Most of them $(65.22 \%$; 15/23) had extra-gonad phenotypes; CHARGE or CHARGE-like symptoms accounted for $80.0 \%$ (13/15) cases and other CHD7-related symptoms were seen in $53.3 \%(8 / 15)$ cases. All CHARGE or CHARGE-like features, except for rhombencephalic anomalies and malformation of mediastinal 
TABLE 2 | Pathogenicity analysis of 29 CHD7 variants.

\begin{tabular}{|c|c|c|c|c|c|c|c|c|c|c|c|c|c|c|c|c|c|c|c|c|}
\hline \multirow{2}{*}{$\begin{array}{l}\text { Nucleotide } \\
\text { change }\end{array}$} & \multirow{2}{*}{\multicolumn{2}{|c|}{$\begin{array}{l}\text { Gnom AD (Allele } \\
\text { frequency) }\end{array}$}} & \multirow{2}{*}{$\begin{array}{c}\text { ClinVar } \\
\text { (Interpreted } \\
\text { condition) }\end{array}$} & \multirow{2}{*}{$\begin{array}{c}\text { CHD7 } \\
\text { database } \\
\text { (mutation ID) }\end{array}$} & \multicolumn{2}{|c|}{$\begin{array}{l}\text { Frequency in our } \\
\text { Cohort }\end{array}$} & \multirow[t]{2}{*}{ OR $(95 \% \mathrm{Cl})$} & \multicolumn{2}{|c|}{ Reference (PMID) } & \multirow{2}{*}{$\begin{array}{c}\text { Recurrent } \\
\text { or Novel }\end{array}$} & \multirow{2}{*}{$\begin{array}{c}\text { Parental } \\
\text { origin }\end{array}$} & \multicolumn{7}{|c|}{ In Silico Analysis } & \multirow[t]{2}{*}{ ACMG criteria } & \multirow[t]{2}{*}{ Classification } \\
\hline & & & & & & & & & & & & SIFT & $\begin{array}{c}\text { Polyphen } \\
2\end{array}$ & $\begin{array}{l}\text { SNPs } \\
\& \text { Co }\end{array}$ & $\begin{array}{l}\text { Mutation } \\
\text { assessor }\end{array}$ & MutPred & $\begin{array}{l}\text { Splice site } \\
\text { score } \\
\text { calculation }\end{array}$ & $\begin{array}{c}\text { Splice } \\
\text { Al }\end{array}$ & & \\
\hline $\begin{array}{l}\text { IVS10: } \\
\text { c 283551G>A }\end{array}$ & - & - & - & - & 0.0031 & $(1 / 327)$ & ' & - & - & Novel & NA & - & - & - & - & - & + & + & $\begin{array}{l}\text { PVS1; PM2; } \\
\text { PVF PPO }\end{array}$ & P \\
\hline $\begin{array}{l}\text { IVS } 23: \\
\text { c. } 5210+3 A>G\end{array}$ & - & - & - & M726 & 0.0031 & $(1 / 327)$ & , & - & - & - & $\mathrm{NA}$ & - & - & - & - & - & + & + & $\begin{array}{l}\text { PVS1; PM2; } \\
\text { PP5; PP3 }\end{array}$ & P \\
\hline $\begin{array}{l}\text { IVS37: } \\
\text { c.7972-1G>C }\end{array}$ & - & - & - & - & 0.0031 & $(1 / 327)$ & ' & - & - & Novel & NA & - & - & - & - & - & + & + & PVS; PM2; PP3 & P \\
\hline Ex2: c.409 T>G & 0.000121 & $(34 / 280358)$ & $\begin{array}{l}\text { CHARGE } \\
\text { syndrome }\end{array}$ & - & 0.0031 & $(1 / 327)$ & $\begin{array}{c}25.29 \\
(3.45-285.30)\end{array}$ & - & - & - & NA & - & - & + & + & + & - & - & PS4; PP5; PP3 & $L P$ \\
\hline EX5: c.2347C>T & 0.0000821 & (23/280310) & $\begin{array}{l}\text { CHARGE } \\
\text { syndrome }\end{array}$ & - & 0.0031 & $(1 / 327)$ & $\begin{array}{c}37.38 \\
(5.03-277.63)\end{array}$ & - & - & - & $\mathrm{NA}$ & - & - & + & + & + & - & - & PS4; PP5; PP3 & $L P$ \\
\hline$E \times 32: 0.6851 \mathrm{G}>\mathrm{A}$ & 0.000004 & (1/249122) & not specified & $\begin{array}{l}\text { Arg2284X } \\
\text { (M49) }\end{array}$ & 0.0031 & $(1 / 327)$ & $\begin{array}{c}764.17 \\
(47.59-12243.89)\end{array}$ & - & - & - & NA & - & + & + & + & + & - & - & PS4; PP5; PP3 & $\llcorner P$ \\
\hline EX34: c.7170 T>G & 0.0000291 & (7/240698) & $\begin{array}{l}\text { CHARGE } \\
\text { syndrome }\end{array}$ & & 0.0031 & $(1 / 327)$ & $\begin{array}{c}105.47 \\
(47.59-12243.89)\end{array}$ & - & - & Novel & NA & - & + & + & + & + & - & - & PS4; PP5; PP3 & $L P$ \\
\hline$E X 4: 02214 A>C$ & - & - & - & - & 0.0062 & $(2 / 237)$ & / & - & - & - & NA & - & - & + & + & + & - & - & PM2; BP4 & us1 \\
\hline EX10: c.2831 G>A & 0.000623 & $\begin{array}{l}(174 / \\
279338)\end{array}$ & $\begin{array}{l}\text { CHARGE } \\
\text { syndrome; } \\
\text { HH (likely } \\
\text { behign) }\end{array}$ & M499 & 0.0031 & $(1 / 327)$ & $\begin{array}{c}4.92 \\
(47.59-12243.89)\end{array}$ & 21158681 & $\begin{array}{c}1 / 642 \\
\text { (CHARGE) }\end{array}$ & - & NA & - & - & + & + & + & - & - & PS4; BP6; PP3 & us1 \\
\hline EX31: $c .6703 \mathrm{~A}>\mathrm{C}$ & $5.66 \mathrm{E}-06$ & (1/1/76564) & - & - & 0.0031 & (2/327) & $\begin{array}{c}1086.55 \\
(98.28-12013.78)\end{array}$ & - & - & Novel & NA & - & - & + & + & + & - & - & PS4; BP4 & us 1 \\
\hline EX15: c.3752 G>T & - & - & - & $\begin{array}{l}\text { Cyst251Arg } \\
\text { (M1014) }\end{array}$ & 0.0031 & $(1 / 327)$ & ' & - & - & - & NA & + & + & + & + & + & - & - & $\begin{array}{l}\text { PM2; } \\
\text { PM5; PP3 }\end{array}$ & us2 \\
\hline EX9: c.2656 C>T & - & - & - & - & 0.0031 & $(1 / 327)$ & 1 & 25077900 & $\begin{array}{c}1 / \\
313(H H)\end{array}$ & - & NA & + & + & + & + & + & - & - & PM2; PP3; PP5 & us2 \\
\hline EX19: c.4516 $\mathrm{G}>\mathrm{A}$ & 0.0000265 & $(7 / 264564)$ & - & - & 0.0062 & (2/327) & $\begin{array}{c}232.39 \\
(48.09-1122.2)\end{array}$ & - & - & Novel & NA & + & + & + & + & + & - & - & PS4; PP3 & us2 \\
\hline$E X 4: 0.2182 G>A$ & - & - & - & M473 & 0.0031 & $(1 / 327)$ & 1 & 21158681 & $\begin{array}{c}\text { 1/642 } \\
\text { (CHARGE) }\end{array}$ & - & matemal & - & - & + & + & + & - & - & $\begin{array}{l}\text { PS4; PP2; } \\
\text { PP5; PP3 }\end{array}$ & us2 \\
\hline$E \times 31: 0.6353 A>G$ & 0.000111 & (31/280350) & - & $\begin{array}{l}\text { Asn21 18Asp } \\
\text { (M1199.Benign) }\end{array}$ & - & - & $\begin{array}{c}55.65 \\
(48.09-1122.2)\end{array}$ & - & - & - & & - & & + & + & + & - & - & - & us2 \\
\hline EX33: c.7083 G>C & $7.35 \mathrm{E}-06$ & $(2 / 272042)$ & - & - & 0.0062 & $(2 / 327)$ & $\begin{array}{c}837.04 \\
(117.55-5960.42)\end{array}$ & - & - & Novel & NA & - & + & + & + & + & - & - & PS4; PP3 & us2 \\
\hline EX34: c.7358 G>A & - & - & - & - & 0.0031 & $(1 / 327)$ & , & - & - & Novel & Paternal & - & + & + & + & + & - & - & PM2; PP3 & us2 \\
\hline EX38: c.8424 C>A & $8.19 \mathrm{E}-06$ & $(2 / 244278)$ & - & - & 0.0031 & $(1 / 327)$ & $\begin{array}{c}374.66 \\
(33.89-4142.13)\end{array}$ & - & - & Novel & NA & - & - & + & + & + & - & - & PS4; PP3 & us2 \\
\hline 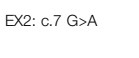 & $4.26 \mathrm{E}-06$ & (1/234534) & - & - & 0.0031 & $(1 / 327)$ & $\begin{array}{c}719.42 \\
(44.90-11526.92)\end{array}$ & - & - & Novel & NA & - & + & + & + & + & - & - & PS4; PP3 & us2 \\
\hline$E X 2: c .120 A>C$ & 0.0000121 & (3/248208) & - & - & 0.0031 & $(1 / 327)$ & $\begin{array}{c}253.78 \\
(26.33-2446.27)\end{array}$ & - & - & Novel & NA & - & + & + & + & + & - & - & PS4; PP3 & us2 \\
\hline EX9: 0.2615 T>C & 0.0000379 & $(9 / 237620)$ & - & - & 0.0031 & $(1 / 327)$ & $\begin{array}{c}80.99 \\
(10.34-641.07)\end{array}$ & - & - & Novel & NA & - & + & + & + & + & - & - & PS4; PP3 & us2 \\
\hline Ex9: c.2662 A>G & 0.0000252 & $(7 / 277580)$ & - & - & 0.0031 & $(1 / 327)$ & $\begin{array}{c}121.64 \\
(14.92-991.45)\end{array}$ & - & - & Novel & NA & - & + & + & + & + & - & - & PS4; PP3 & us2 \\
\hline EX3: c.1853 A>G & - & - & - & - & 0.0031 & $(1 / 327)$ & 1 & - & - & Novel & paternal & - & + & + & + & + & - & - & PM2; PP3 & us2 \\
\hline EX4: c.2219 A>G & - & - & - & - & 0.0031 & $(1 / 327)$ & , & 22033296 & $\begin{array}{c}\text { 1/50 } \\
\text { (CHARGE) }\end{array}$ & Novel & $\mathrm{NA}$ & - & + & + & + & + & - & - & $\begin{array}{l}\text { PM2; } \\
\text { PM5; PP3 }\end{array}$ & us2 \\
\hline EX9: c.2690 G>C & - & - & - & - & 0.0031 & $(1 / 327)$ & 1 & - & - & Novel & NA & - & - & + & + & + & - & - & PM2; PP3 & us2 \\
\hline EX16: c.3932 T>C & - & - & - & - & 0.0031 & $(1 / 327)$ & ' & - & - & Novel & maternal & + & + & + & + & + & - & - & PM2; РP3 & us2 \\
\hline EX23: c.5095 A>G & - & - & - & - & 0.0031 & $(1 / 3$ & 1 & - & - & Novel & matemal & + & - & - & + & + & - & - & PM2; PP3 & us2 \\
\hline$E \times 31: c .6368 \mathrm{C}>\mathrm{G}$ & - & - & - & - & 0.0031 & $(1 / 327)$ & 1 & - & - & Novel & matemal & + & - & + & + & + & - & - & PM2; PP5; PP3 & us2 \\
\hline EX33: c.6980 T>G & - & - & - & - & 0.0031 & $(1 / 327)$ & 1 & - & - & Novel & NA & + & + & + & + & + & - & - & PM2; PP3 & us2 \\
\hline EX24: c5227 C>T & - & - & - & - & 0.0031 & $(1 / 327)$ & i & - & - & Novel & $\mathrm{NA}$ & + & + & + & + & + & - & - & PM2; PP3 & us2 \\
\hline
\end{tabular}

*, pathogenic $\geq 3$; P, pathogenic; LP, likely pathogenic; US1, uncertain significance with paradoxical evidence; US2, uncertain significance without enough evidence; US, uncertain significance; NA, not available. 

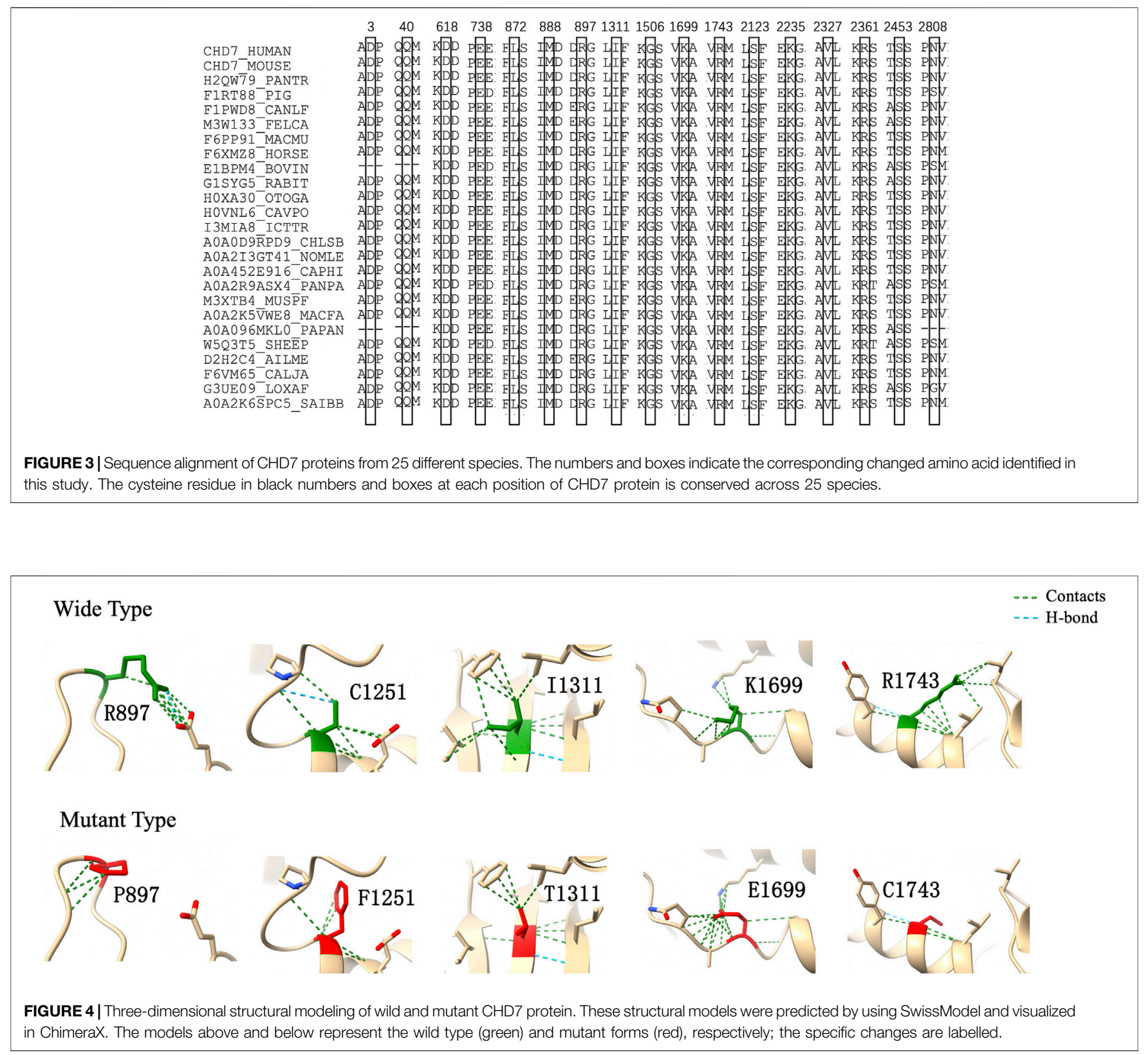

viscera, occurred in our patients; hearing loss and high myopia were the most frequent ones $(5 / 10 ; 50 \%)$. Patient $21(\mathrm{P} 21$ in Table 3) was re-diagnosed as having typical CHARGE syndrome based on three major and six minor diagnostic items. Other CHD7-related symptoms included high arched palate $(50 \% ; 4 / 8)$, spinal malformation $(25 \% ; 2 / 8)$, digestive system dysfunction $(25 \% ; 2 / 8)$, secondary hypothyroidism $(12.5 \% ; 1 / 8)$, six fingers in the left hand $(12.5 \% ; 1 / 8)$, inguinal hernia $(12.5 \% ; 1 / 8)$, micrognathism $(12.5 \% ; 1 / 8)$, widened palpebral fissure $(12.5 \% ; 1 / 8)$, epicanthus $(12.5 \% ; 1 / 8)$, and short philtrum fish mouth (12.5\%; $1 / 8)$.

Occurrence of extragonadal symptoms correlated with the pathogenicity of variants. All CHH patients with P or LP variant manifested CHARGE-related symptoms or (and) other CHD7related manifestations besides $\mathrm{CHH}$. For patients carrying US1 and US2 variants, the frequency of CHARGE-related symptoms or others was $0 \%(2 / 2)$ and $64.71 \%(11 / 17)$, respectively.

\section{DISCUSSION}

We identified 30 types of CHD7 RSVs with a detection rate of $11.01 \%$ [ $8.11 \%$ in $\mathrm{nCHH}(12 / 148)$ and $13.41 \%$ in $\mathrm{KS}(24 / 179)]$ in our series of $327 \mathrm{CHH}$ patients. P or LP variants accounted for $23.33 \%$ (7/30) cases. Previous studies on the pathogenic role of CHD7 RSVs mainly focused on CHARGE syndrome, In 2008, 
TABLE 3 | CHD7-related characteristics analysis in $23 \mathrm{CHH}$ patients harboring $C H D 7$ rare variants.

\begin{tabular}{|c|c|c|c|c|c|c|c|}
\hline \multirow[t]{2}{*}{ Patients } & \multirow[t]{2}{*}{ Dignosis } & \multirow[t]{2}{*}{ Sex } & \multirow{2}{*}{$\begin{array}{l}\text { Nucleotide } \\
\text { Change }\end{array}$} & \multirow[t]{2}{*}{ Other variants } & \multicolumn{2}{|c|}{ CHARGE syndrome related symptoms } & \multirow[t]{2}{*}{ Others } \\
\hline & & & & & Major & $\begin{array}{c}\text { Minor or } \\
\text { CHARGE-like symptoms }\end{array}$ & \\
\hline \multicolumn{8}{|c|}{ Patient with $\mathrm{P}$ variants } \\
\hline P20 & $\mathrm{nCHH}$ & $\mathrm{M}$ & c. $2835+1 G>G$ & - & UA & $\begin{array}{l}\text { Deafness; } \\
\text { Abnormal external ear } \\
\text { Intellectual Disability } \\
\text { Malformation of mediastinal } \\
\text { organs (heart) }\end{array}$ & $\begin{array}{l}\text { Scoliosis } \\
\text { Digestive system } \\
\text { disfuction } \\
- \\
\text { - }\end{array}$ \\
\hline P21 & $\mathrm{nCHH}$ & $M$ & c. $5210+3 A>G$ & - & $\begin{array}{l}\text { Chanal atresia; Coloboma; } \\
\text { semicircular canal anomalies }\end{array}$ & $\begin{array}{l}\text { High Myopia Neuro sensory } \\
\text { deafness } \\
\text { Facial asymmetry } \\
\text { Abnormal external ear } \\
\text { Intellectual Disability } \\
\text { Malformation of mediastinal } \\
\text { organs (heart) } \\
\text { Microphthalmia }\end{array}$ & $\begin{array}{l}\text { Scoliosis } \\
- \\
- \\
-\end{array}$ \\
\hline \multicolumn{8}{|c|}{ Patient with LP variants } \\
\hline P25 & KS & $\mathrm{F}$ & c. $6851 \mathrm{G}>\mathrm{A}$ & - & - & $\begin{array}{l}\text { Deafness } \\
\text { Abnormal external ear } \\
\text { Growth hormone deficiency }\end{array}$ & High palate \\
\hline P8 & $\mathrm{nCHH}$ & M & c.7170 T > G & - & - & Intellectual Disability & - \\
\hline \multicolumn{8}{|c|}{ Patients with US1 variants } \\
\hline P32 & KS & M & c.2214 A > C & - & - & - & - \\
\hline P2 & $\mathrm{nCHH}$ & M & c. $6703 \mathrm{~A}>\mathrm{C}$ & - & - & - & - \\
\hline \multicolumn{8}{|c|}{ Patients with US2 variants } \\
\hline P14 & KS & $\mathrm{M}$ & $\begin{array}{l}\text { c. } 2656 \mathrm{C}>\mathrm{T} \\
\text { c. } 4516 \mathrm{G}>\mathrm{A}\end{array}$ & - & - & - & Inguinal hernia \\
\hline P34 & $\mathrm{KS}$ & $\mathrm{M}$ & c. $4516 \mathrm{G}>\mathrm{A}$ & - & - & - & $\begin{array}{l}\text { High palate; polysyndactyly } \\
\text { (left hand) }\end{array}$ \\
\hline P33 & KS & M & c.7358 G > A & - & - & Growth hormone deficiency & - \\
\hline P35 & KS & $\mathrm{M}$ & c. $120 \mathrm{~A}>\mathrm{C}$ & - & - & $\begin{array}{l}\text { Micrognathism } \\
\text { Widened palpebral fissure } \\
\text { Epicanthus } \\
\text { Short philtrum fish mouth }\end{array}$ & High palate \\
\hline P16 & KS & $\mathrm{M}$ & c. $1853 \mathrm{~A}>\mathrm{G}$ & $\begin{array}{l}\text { GNRH1, } \\
\text { p.Gly34Arg }\end{array}$ & - & - & - \\
\hline P24 & $\mathrm{nCHH}$ & $\mathrm{M}$ & c. $2219 A$ > G & - & - & $\begin{array}{l}\text { High } \\
\text { Myopia }\end{array}$ & - \\
\hline P6 & KS & $\mathrm{M}$ & c.3932 T > C & - & - & - & $\begin{array}{l}\text { Secondary } \\
\text { hypothyroidism }\end{array}$ \\
\hline P31 & KS & $\mathrm{F}$ & c.6368 C > G & - & - & - & - \\
\hline P7 & KS & $M$ & c.5227 C > T & - & - & - & - \\
\hline P36 & $\mathrm{KS}$ & $\mathrm{M}$ & c.5227 C > T & - & - & - & - \\
\hline P4 & KS & $\mathrm{M}$ & c.6980 T > G & - & - & $\begin{array}{l}\text { Deafness } \\
\text { High Myopia }\end{array}$ & - \\
\hline P18 & KS & $\mathrm{M}$ & c. $2615 \mathrm{~T}>\mathrm{C}$ & - & - & $\begin{array}{l}\text { Malformation of mediastinal } \\
\text { organs (heart) } \\
\text { Growth hormone deficiency } \\
\text { High Myopia }\end{array}$ & - \\
\hline P17 & $\mathrm{nCHH}$ & $\mathrm{M}$ & c. $4516 \mathrm{G}>\mathrm{A}$ & - & - & $\begin{array}{l}\text { Deafness } \\
\text { Abnormal external ear } \\
\text { Growth hormone deficiency }\end{array}$ & - \\
\hline P5 & KS & $\mathrm{M}$ & c. $7083 \mathrm{G}>\mathrm{C}$ & - & - & - & - \\
\hline P23 & $\mathrm{nCHH}$ & $\mathrm{M}$ & c. 2662 A > G & - & - & $\begin{array}{l}\text { High } \\
\text { Myopia }\end{array}$ & $\begin{array}{l}\text { Digestive system } \\
\text { disfuction }\end{array}$ \\
\hline P29 & KS & $\mathrm{M}$ & c. $8424 \mathrm{C}>\mathrm{A}$ & - & - & - & - \\
\hline P26 & KS & $\mathrm{M}$ & c. $7 \mathrm{G}>\mathrm{A}$ & - & - & Deafness & - \\
\hline
\end{tabular}

KS, kallmann syndrome; $n C H H$, normosmic congenital hypogonadotropic hypogonadism; $M$, male; F, female; UA, unavailable.

Kim et al. (2008) first showed that Chd7 mRNA expressed in the $\mathrm{CHH} / \mathrm{KS}$-relevant organs of rats (including migratory and postmigratory GnRH neuron, olfactory bulb, pituitary, and hypothalamus), and the pathogenic variants can exist in both $\mathrm{nCHH}$ and $\mathrm{KS}$ without CHARGE-related phenotype. Thus, the triple correlation among $\mathrm{CHD}, \mathrm{CHH}$, and $\mathrm{CHARGE}$ started to 
gain importance. Our CHD7 detection rate was consistent with that reported in previous four studies, which explored $\mathrm{CHD} 7$ variants in $\mathrm{CHH}$ patients, with detection rates of $16 \%(18 / 116)$ (Xu et al., 2018), 16\% (8/50) (Gonçalves et al., 2019), 10.2\% (18/ 177) (Li et al., 2020), and 6\% (6/101) 9

Our variants distributed throughout the CHD7 gene and protein region, and tended to cluster around exons 2,4 , and 8 of the gene model and three regions without known functional domain of the protein model, whereas pathogenic or likely pathogenic RSVs showed no "hot-spot" tendency. To further explore the potential function of the unknown protein regions (amino acids 1-202; 576-801; 1,404-2,564), we looked them up in the InterPro website and found no conserved regions. This may be an evidence for the hypothesis that $C H D 7$ variants in humans can lead to a continuous phenotype spectrum, and $\mathrm{CHH}$ is just a milder manifestation than CHARGE syndrome (Kim et al., 2008). The report byBergman et al. (2012) also supports this hypothesis. In their CHARGE syndrome patients, pathogenic missense mutations were mainly found in the middle of the CHD7 gene, where functional domains clustered.

We annotated the variants manually according to the ACMG guideline and further classified US variants into US1 and US2, which may be suggestive for variant classification in the future. Most of the variants $(66.67 \% ; 20 / 30)$ were classified as US. Four variants (E738D, A2225T, K2235Q, and R944H) belonged to US1 (need to be further confirmed by functional experiments) and 20 variants (G1506S, N2118S, R2361S, N2808K, D3N, Q40H, I872T, S2453N, D618G, D740G, R897P, I1311T, K1699E, S2123C, V2327G, R1743C, D728N, C1251F, R886W, and M888) belonged to US2 (reclassification may be required with additional global evidence obtained through studies in the future).

One patient (P21 in Table 3) was re-diagnosed as having CHARGE syndrome in our $\mathrm{CHH}$ series. Similarly, the study by $\mathrm{Xu}$ et al. reclassified 3 out of 17 patients. Except for rhombencephalic anomalies and malformation of the mediastinal viscera, all CHARGE syndrome diagnostic items were noted in our $\mathrm{CHH}$ patients with $\mathrm{CHD} 7$ variants, and hearing loss $(5 / 10 ; 50 \%)$ and high myopia $(5 / 10 ; 50 \%)$ were the most common ones. However, in the study by $\mathrm{Wu}$ et al., hearing loss $(6 / 18,33.3 \%)$ and ear deformities $(3 / 18,16.7 \%)$, the two diagnostic CHARGE features, were significantly frequent in patients with $\mathrm{CHD} 7$ variants. As for other symptoms besides $\mathrm{CHH}$ and $\mathrm{CHARGE}$, we first reported polysyndactyly, inguinal hernia, small chin and short philtrum fish mouth in $\mathrm{CHH}$ patients with $C H D 7$ variants, and all these phenotypes existed in the same patient (P35 in Table 3), who carried the variant Q40H.

No consensus on the genotype-phenotype correlation exists. In our study, we observed a correlation between variant pathogenicity and extragonadal symptoms. Extragonadal symptoms were present more commonly in patients with $\mathrm{P}$ or LP variants $(4 / 4,100.0 \%)$ than in patients with US1 $(0 / 2,0 \%)$ or US2 (11/17, 64.71\%) variants. In 2017, Xu et al. (2018) first evaluated CHARGE syndrome in $166 \mathrm{CHH}$ patients, and detailed phenotyping in 17 patients revealed that $80 \%(4 / 5)$ of patients with P or LP variants showed multiple CHARGE features versus
8\% (1/12) with nonpathogenic (US, B, and LB) variants. However, in studies by Jongmans et al. (2006) and Bergman et al. (2011), no genotype-phenotype correlation existed in CHARGE syndrome patients. Due to the small sample of patients with $\mathrm{P}$ and LP variants and the lack of awareness of the true classification of US variants, we could not ascertain the difference between each subgroup. Therefore, even though specific clinical manifestations can provide us with information on genetic hits, this finding needs to be interpreted with caution.

The main strengths of our study are as follows: (1) This study systematically analyzed genotype, phenotype, and their correlation in a series of $\mathrm{CHH}$ patients with $\mathrm{CHD} 7$ gene variants; (2) 19 novel variants were reported to expand the genotype spectrum; (3) Variants were classified according to the ACMG guideline, and the US type variants were further classified into US1 and US2, which provide detailed evidence for future studies; (4) Our study showed a genotype-phenotype correlation tendency, namely P or LP RSVs were more likely to cause syndromic $\mathrm{CHH}$.

Besides, there are also some limitations in our study that need to be mentioned. (1) Owing to partial information provided by the probands' family, evidence for the genotype-phenotype cosegregation was unavailable, which could elaborate the contribution of $\mathrm{CHD} 7$ variants to these phenotypes besides $\mathrm{CHH}$; (2) This comprehensive clinical analysis was retrospective, and loss of follow-up existed; therefore, the prevalence of phenotypes besides $\mathrm{CHH}$ may be underestimated in $\mathrm{CHH}$ patients with $\mathrm{CHD} 7$ variants. In future studies, it would be necessary to collect information on the probands' family blood tests and perform comprehensive physical examination.

\section{CONCLUSION}

19 novel $\mathrm{CHD} 7$ variants reported herein expand the existing variant spectrum. $\mathrm{P}$ or LP RSVs are more likely to cause syndromic $\mathrm{CHH}$. For $\mathrm{CHH}$ patients carrying CHD7 RSVs, early detailed genotyping and phenotyping can help clinical diagnosis and therapy.

\section{DATA AVAILABILITY STATEMENT}

The datasets presented in this study can be found in online repositories. The names of the repository/repositories and accession number(s) can be found in the article/Supplementary Material.

\section{ETHICS STATEMENT}

The studies involving human participants were reviewed and approved by the Ethics Committee for Human Research of Peking Union Medical College Hospital. The patients/ participants provided their written informed consent to participate in this study. Written informed consent was 
obtained from the individual(s) for the publication of any potentially identifiable images or data included in this article.

\section{AUTHOR CONTRIBUTIONS}

BS carried out the study and wrote the manuscript. XW and JM provided patients. ZZ and WZ revised the manuscript. $\mathrm{MN}$ and $\mathrm{XW}$ designed the study and revised the manuscript.

\section{FUNDING}

This work was supported by the National Natural Science Foundation of China (Grant Nos. 81771576 and 81971375)

\section{REFERENCES}

Adzhubei, I. A., Schmidt, S., Peshkin, L., Ramensky, V. E., Gerasimova, A., Bork, P., et al. (2010). A Method and Server for Predicting Damaging Missense Mutations. Nat. Methods 7, 248-249. doi:10.1038/nmeth0410-248

Balasubramanian, R., Choi, J.-H., Francescatto, L., Willer, J., Horton, E. R., Asimacopoulos, E. P., et al. (2014). Functionally compromisedCHD7alleles in Patients with Isolated GnRH Deficiency. Proc. Natl. Acad. Sci. USA 111, 17953-17958. doi:10.1073/pnas.1417438111

Bartels, C. F., Scacheri, C., White, L., Scacheri, P. C., and Bale, S. (2010). Mutations in the CHD7 Gene: the Experience of a Commercial Laboratory. Genet. Test. Mol. Biomarkers 14, 881-891. doi:10.1089/gtmb.2010.0101

Bergman, J. E. H., Janssen, N., Hoefsloot, L. H., Jongmans, M. C. J., Hofstra, R. M. W., and van Ravenswaaij-Arts, C. M. A. (2011). CHD7 Mutations and CHARGE Syndrome: the Clinical Implications of an Expanding Phenotype. J. Med. Genet. 48, 334-342. doi:10.1136/jmg.2010.087106

Bergman, J. E. H., Janssen, N., van der Sloot, A. M., de Walle, H. E. K., Schoots, J., Rendtorff, N. D., et al. (2012). A Novel Classification System to Predict the Pathogenic Effects of CHD7 Missense Variants in CHARGE Syndrome. Hum. Mutat. 33, 1251-1260. doi:10.1002/humu.22106

Bhagavath, B., Podolsky, R. H., Ozata, M., Bolu, E., Bick, D. P., Kulharya, A., et al. (2006). Clinical and Molecular Characterization of a Large Sample of Patients with Hypogonadotropic Hypogonadism. Fertil. Sterility 85, 706-713. doi:10.1016/j.fertnstert.2005.08.044

Bilan, F., Legendre, M., Charraud, V., Manière, B., Couet, D., and GilbertDussardier, B. (2012). Complete Screening of 50 Patients with CHARGE Syndrome for Anomalies in the CHD7 Gene Using a Denaturing HighPerformance Liquid Chromatography-Based Protocol: New Guidelines and a Proposal for Routine Diagnosis. J. Mol. Diagn. 14, 46-55. doi:10.1016/ j.jmoldx.2011.08.003

Bland, J. M., and Altman, D. G. (2000). Statistics Notes: The Odds Ratio. BMJ 320, 1468. doi:10.1136/bmj.320.7247.1468

Boehm, U., Bouloux, P.-M., Dattani, M. T., de Roux, N., Dodé, C., Dunkel, L., et al. (2015). Expert Consensus Document: European Consensus Statement on Congenital Hypogonadotropic Hypogonadism-Ppathogenesis, Diagnosis and Treatment. Nat. Rev. Endocrinol. 11, 547-564. doi:10.1038/nrendo.2015.112

Dunnen, J. T. d., and Antonarakis, S. E. (2000). Mutation Nomenclature Extensions and Suggestions to Describe Complex Mutations: a Discussion. Hum. Mutat. 15, 7-12. doi:10.1002/(sici)1098-1004(200001)15:1<7:aid-humu4>3.0.co;2-n

Gonçalves, C. I., Patriarca, F. M., Aragüés, J. M., Carvalho, D., Fonseca, F., and Martins, S. (2019). High Frequency of CHD7 Mutations in Congenital Hypogonadotropic Hypogonadism. Sci. Rep. 9, 1597. doi:10.1038/s41598018-38178-y

Herbison, A. E. (2016). Control of Puberty Onset and Fertility by GonadotropinReleasing Hormone Neurons. Nat. Rev. Endocrinol. 12, 452-466. doi:10.1038/ nrendo.2016.70 and the Beijing Municipal Natural Science Foundation (7202151 and 7212080).

\section{ACKNOWLEDGMENTS}

We thank the subjects and their family members for their participation in the research.

\section{SUPPLEMENTARY MATERIAL}

The Supplementary Material for this article can be found online at: https://www.frontiersin.org/articles/10.3389/fgene.2021.770680/ full\#supplementary-material

Jaganathan, K., Kyriazopoulou Panagiotopoulou, S., McRae, J. F., Darbandi, S. F., Knowles, D., Li, Y. I., et al. (2019). Predicting Splicing from Primary Sequence with Deep Learning. Cell 176, 535-548. doi:10.1016/j.cell.2018.12.015

Jongmans, M. C. J., Admiraal, R. J., van der Donk, K. P., Vissers, L. E. L. M., Baas, A. F., Kapusta, L., et al. (2006). CHARGE Syndrome: the Phenotypic Spectrum of Mutations in the CHD7 Gene. J. Med. Genet. 43, 306-314. doi:10.1136/ jmg.2005.036061

Kim, H.-G., Kurth, I., Lan, F., Meliciani, I., Wenzel, W., Eom, S. H., et al. (2008). Mutations in CHD7, Encoding a Chromatin-Remodeling Protein, Cause Idiopathic Hypogonadotropic Hypogonadism and Kallmann Syndrome. Am. J. Hum. Genet. 83, 511-519. doi:10.1016/j.ajhg.2008.09.005

Laitinen, E.-M., Vaaralahti, K., Tommiska, J., Eklund, E., Tervaniemi, M., Valanne, L., et al. (2011). Incidence, Phenotypic Features and Molecular Genetics of Kallmann Syndrome in Finland. Orphanet J. Rare Dis. 6, 41. doi:10.1186/17501172-6-41

Li, B., Krishnan, V. G., Mort, M. E., Xin, F., Kamati, K. K., Cooper, D. N., et al. (2009). Automated Inference of Molecular Mechanisms of Disease from Amino Acid Substitutions. Bioinformatics 25, 2744-2750. doi:10.1093/bioinformatics/ btp528

Li, J.-D., Wu, J., Zhao, Y., Wang, X., Jiang, F., Hou, Q., et al. (2020). Phenotypic Spectrum of Idiopathic Hypogonadotropic Hypogonadism Patients with CHD7 Variants from a Large Chinese Cohort. J. Clin. Endocrinol. Metab. 105, 1515-1526. doi:10.1210/clinem/dgz182

Marcos, S., Sarfati, J., Leroy, C., Fouveaut, C., Parent, P., Metz, C., et al. (2014). The Prevalence ofCHD7Missense versus Truncating Mutations Is Higher in Patients with Kallmann Syndrome Than in Typical CHARGE Patients. J. Clin. Endocrinol. Metab. 99, E2138-E2143. doi:10.1210/jc.2014-2110

Martin, D. M. (2010). Chromatin Remodeling in Development and Disease: Focus on CHD7. Plos Genet. 6, e1001010. doi:10.1371/journal.pgen.1001010

Nie, M., Yu, B., Chen, R., Sun, B., Mao, J., Wang, X., et al. (2021). Novel Rare Variants in FGFR1 and Clinical Characteristics Analysis in a Series of Congenital Hypogonadotropic Hypogonadism Patients. Clin. Endocrinol. 95, 153-162. doi:10.1111/cen.14436

Oliveira, L. M. B., Seminara, S. B., Beranova, M., Hayes, F. J., Valkenburgh, S. B., Schipani, E., et al. (2001). The Importance of Autosomal Genes in Kallmann Syndrome: Genotype-Phenotype Correlations and Neuroendocrine Characteristics1. J. Clin. Endocrinol. Metab. 86, 1532-1538. doi:10.1210/ jcem.86.4.7420

Reva, B., Antipin, Y., and Sander, C. (2011). Predicting the Functional Impact of Protein Mutations: Application to Cancer Genomics. Nucleic Acids Res. 39, e118. doi:10.1093/nar/gkr407

Riecher-Rössler, A. (2017). Oestrogens, Prolactin, Hypothalamic-PituitaryGonadal axis, and Schizophrenic Psychoses. Lancet Psychiatry 4, 63-72. doi:10.1016/S2215-0366(16)30379-0

Schwarz, J. M., Rödelsperger, C., Schuelke, M., and Seelow, D. (2010). MutationTaster Evaluates Disease-Causing Potential of Sequence Alterations. Nat. Methods 7, 575-576. doi:10.1038/nmeth0810-575 
Seminara, S. B., Hayes, F. J., and Crowley, W. F. (1998). GonadotropinReleasing Hormone Deficiency in the Human (Idiopathic Hypogonadotropic Hypogonadism and Kallmann's Syndrome): Pathophysiological and Genetic Considerations. Endocr. Rev. 19, 521-539. doi:10.1210/edrv.19.5.0344

Sim, N.-L., Kumar, P., Hu, J., Henikoff, S., Schneider, G., and Ng, P. C. (2012). SIFT Web Server: Predicting Effects of Amino Acid Substitutions on Proteins. Nucleic Acids Res. 40, W452-W457. doi:10.1093/nar/gks539

Stamou, M. I., and Georgopoulos, N. A. (2018). Kallmann Syndrome: Phenotype and Genotype of Hypogonadotropic Hypogonadism. Metabolism 86, 124-134. doi:10.1016/j.metabol.2017.10.012

Verloes, A. (2005). Updated Diagnostic Criteria for CHARGE Syndrome: a Proposal. Am. J. Med. Genet. 133A, 306-308. doi:10.1002/ajmg.a.30559

Vissers, L. E. L. M., van Ravenswaaij, C. M. A., Admiraal, R., Hurst, J. A., de Vries, B. B. A., Janssen, I. M., et al. (2004). Mutations in a New Member of the Chromodomain Gene Family Cause CHARGE Syndrome. Nat. Genet. 36, 955-957. doi:10.1038/ng1407

Xu, C., Cassatella, D., van der Sloot, A. M., Quinton, R., Hauschild, M., De Geyter, C., et al. (2018). Evaluating CHARGE Syndrome in Congenital
Hypogonadotropic Hypogonadism Patients Harboring CHD7 Variants. Genet. Med. 20, 872-881. doi:10.1038/gim.2017.197

Conflict of Interest: The authors declare that the research was conducted in the absence of any commercial or financial relationships that could be construed as a potential conflict of interest.

Publisher's Note: All claims expressed in this article are solely those of the authors and do not necessarily represent those of their affiliated organizations, or those of the publisher, the editors and the reviewers. Any product that may be evaluated in this article, or claim that may be made by its manufacturer, is not guaranteed or endorsed by the publisher.

Copyright (c) 2022 Sun, Wang, Mao, Zhao, Zhang, Nie and Wu. This is an openaccess article distributed under the terms of the Creative Commons Attribution License (CC BY). The use, distribution or reproduction in other forums is permitted, provided the original author(s) and the copyright owner(s) are credited and that the original publication in this journal is cited, in accordance with accepted academic practice. No use, distribution or reproduction is permitted which does not comply with these terms. 